\title{
The Impact of Orang Asli Students' Learning Styles on Their Achievement of Meaningful Learning
}

\author{
Intan Farahana Kamsin, Fariza Khalid, Nor Syazwani Mat Salleh, Analisa Hamdan, \\ Siti Zuraida Abdul Manaf
}

\begin{abstract}
Orang Asli was the original people, who are majority of them located in peninsular Malaysia. They are facing a difficulty in education since less meaningful environment created for them which integrate their preferable learning styles. Additionally previous studies show that Orang Asli students had low motivation level in learning since less condusive education enviroment developed for them. Thus, it is important that teachers have a sound understanding of their students' different learning styles. The significance of understanding students' learning styles also applies to Orang Asli students. However, perhaps due to their isolation, this group's need for meaningful education has not been widely studied. Consequently, a lack of literature on their learning styles is apparent. This study aims to measure whether the learning styles positively affect meaningful learning among Orang Asli students. A quantitative research was conducted which involved 72 Orang Asli students from an Orang Asli community in Selangor, Malaysia. The data was collected using the Meaningful Hybrid E-Training Instruments. Data was analyzed with the SmartPLS 3.0 software. The research findings show that the instrument is highly reliable and valid. Moreover, it has been discovered that Orang Asli students' learning styles have a positive effect upon their meaningful learning. This study demonstrated that teaching and learning activities catering to these students' different learning styles allow meaningful learning to be achieved more successfully. Therefore, teachers are highly encouraged to take into account the various learning styles of Orang Asli students in their teaching in order to help them achieve meaningful learning.
\end{abstract}

Keywords : Orang Asli, Learning Style, Meaningful Learning

Revised Manuscript Received on July 10, 2020.

* Correspondence Author

Intan Farahana Kamsin, Department of Technology Management, Faculty of Technology, Asia Pacific University of Technology \& Innovation, Technology Park Malaysia, Bukit Jalil, 57000 Kuala Lumpur, Malaysia. E-mail: intan.farahana@apu.edu.my

Fariza Khalid*, Centre of Teaching and Learning Innovations, Faculty of Education, Universiti Kebangsaan Malaysia, 43600 Bangi, Selangor, Malaysia. E-mail: fariza.khalid@ukm.edu.my

Nor Syazwani Mat Salleh, Department of Art and Design, Faculty of Art, Computing and Creative Industry, Sultan Idris Education University, 35900 Tanjung Malim, Perak Darul Ridzuan, Malaysia. E-mail: syazwani.ms@fskik.upsi.edu.my

Analisa Hamdan, Faculty of Human Development, Sultan Idris Education University, 35900 Tanjung Malim, Perak Darul Ridzuan, Malaysia. E-mail: analisa.hamdan@gmail.com

Siti Zuraida Abdul Manaf, Faculty of Education, Universiti Kebangsaan Malaysia, 43600 Bangi, Selangor, Malaysia. E-mail: cikgusitizuraida@gmail.com

(C) The Authors. Published by Blue Eyes Intelligence Engineering and Sciences Publication (BEIESP). This is an open access article under the CC BY-NC-ND license (http://creativecommons.org/licenses/by-nc-nd/4.0/)

\section{INTRODUCTION}

Orang Asli people constitute one of the components of the various races in Malaysia. From the point of modern society, Orang Asli people are separated and isolated from the mainstream of national development [1]. They are differentiated by language, culture, lifestyle and physical characteristics. When compared to other ethnic groups, the Orang Asli group still lags behind in many aspects. These include education, economic and social aspects [2]. Before the establishment of the Department of Orang Asli Affair in this country, Orang Asli people, particularly those living in rural areas, had never received formal education. Orang Asli, are well known for their skills in hunting, fishing, and gathering of forest products. This situation seems to be perfectly suited to their way of life, which is based on a sylvan economy [3] [4]. However, these skills remain insufficient for the younger generation, as having an adequate education will be more crucial to improve their standard of living in the future. Lifelong learning is an education democratization process which includes the acquisition of knowledge, skills and competence, formally or informally based on experience and training. Lifelong learning is essential to increase the added value to the community and is considered as the main contributor to productivity and the development of the nation [5] [6]. This aspiration will not be achievable without active involvement from the youth, as they will lead the nation and the education process [7] [8] [9]. However, these aspirations are unlikely to be achieved if the issue of school dropout among these students still occurs. One of the factors contributing to the increase in the dropout rate among Orang Asli students is due to the fact that these students are unable to appreciate the learning they experienced in school as something meaningful and significant to their lives. Meaningful learning is an important aspect that helps students constructs their understanding of information or knowledge, which they acquire [10]. Meaningful learning produces a meaningful understanding which provides the Orang Asli students with an experience on which they can reflect upon what they have learned [11]. When meaningful learning is unachievable, the performance of the students decreases. As a consequence, the dropout rate increases among these students [12] [13]. This problem should be seriously addressed so that the progression of Orang Asli students may be on par with the performance of students in regular schools.

Published By:

Blue Eyes Intelligence Engineering

\& Sciences Publication

(C) Convriaht: All riahts reserved

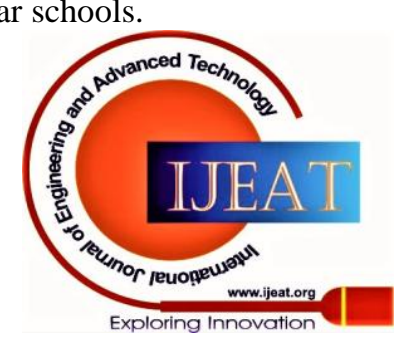




\section{The Impact of Orang Asli Students' Learning Styles on Their Achievement of Meaningful Learning}

Emphasis is given to Orang Asli students because their reading, writing and arithmetic abilities are lower than students from regular schools [14] [15]. Moreover a high dropout rate among them was reported [14]. One effort that can be implemented to overcome this problem is through programs which increase the production of meaningful learning.

This would improve their quality of life and enable them to compete with the outside world [16]. For these reasons, the present study aimed to measure whether learning styles positively affect meaningful learning among Orang Asli students.

\section{RESEARCH HYPOTHESIS}

In the present study, 13 research hypothesis were addressed:

1. The auditory learning styles positively reflect learning styles of Orang Asli students.

2. The phrase learning styles positively reflect learning styles of Orang Asli students.

3. The tactile learning styles positively reflect learning styles of Orang Asli students.

4. The kinesthetic learning styles positively reflect learning styles of Orang Asli students.

5. The visual learning styles positively reflect learning styles of Orang Asli students.

6. The group learning styles positively reflect learning styles of Orang Asli students.

7. The Individual learning styles positively reflect learning styles of Orang Asli students.

8. The objective meaningful learning attribute reflects Orang Asli students' meaningful learning.

9. The active meaningful learning attribute reflects Orang Asli students' meaningful learning.

10. The authentic meaningful learning attribute reflects Orang Asli students' meaningful learning.

11. The cooperative meaningful learning attribute reflects Orang Asli students' meaningful learning.

12. The constructive meaningful learning attribute reflects Orang Asli students' meaningful learning.

13. Learning styles have a positive effect toward meaningful learning among Orang Asli students.

\section{METHODOLOGY}

This study employed a survey method using a meaningful hybrid e-training instrument. The additional attribute 'phrase' for learning styles was included in the instrument development process. Phrase was an emerging theme in a qualitative study done, which aimed to explore the learning styles among Orang Asli students [17]. Furthermore, the instrument was also adapted by changing language and words used. This process was important to ensure that the respondent could understand the meaning of each item. To ensure the validity and reliability of the instrument, a pre study was conducted involving 3 Orang Asli students. This was also to ensure that the language of the items was understandable. This was followed by a pilot study which involved 30 Orang Asli students. The findings of the pilot study were analyzed using the Bond \& FoxSteps 1.0.0 software; a software used in Rasch Measurement Model. Based on four analyses, (i) item reliability (ii) fit item, (iii) item map, and (iv) item dimensionality, the instrument was found to be valid and reliable.

A total of 72 Orang Asli students from an Orang Asli community in Selangor were selected as respondents of this study. Their ages ranged from 12 to 19 years old. The selection of this community was based on three criteria, which were: (i) to have a well-managed organization able to cooperate with the researcher; (ii) to have facilities (hall, library and room) where the research could be conducted; and (iii) a location that was near from the researcher's place to make it cost and time-effective.

\section{FINDINGS}

This section elaborates on the findings regarding the impact of learning styles of Orang Asli students upon their meaningful learning. There are seven attributes of learning styles: tactile, kinesthetic, visual, phrase, auditory, group, and individual. The five attributes of meaningful learning are authentic, active, constructive, cooperative, and objective. The analysis process involved the use of Partial Least Square (PLS) statistical equation modelling (SEM) version 3.0. By using this software, a complete analysis can be conducted for each variable and its related attribute. Measurement scales using PLS are naturally focused on exploration concepts, and is the best choice to elaborate the data [18]. The instruments were distributed to 72 Orang Asli students, and a 500-time resampling (bootstrapping) process was carried out to ensure the significance of the data analyzed. It is recommended that the values used in the resampling process should be higher than the values of respondents involved in a real study [19]. These sections are divided into five, which are (i) item loading values, (ii) construct validity, (iii) convergent validity, (iv) discriminant validity, and (v) hypotheses testing.

\section{A. Item Loadings Value}

Loading values of the item is the first criteria should be consider in a process to identify the variation in an item. It is explained by the construct and is described as the variance extracted from the item [19]. However, there is frequently weaker loading observe especially when newly developed instrument are used [20]. Basically, loadings range between 0.40 and 0.70 should be consider for deleting purpose, when it leads to an increase in the composite reliable. Indicator with very low loadings (below 0.40) should be always eliminated from the instruments [18]. For this purpose, cutoff value used in this study for loadings to be significant was at 0.50 [21]. Table 1 shows the loading values for the indicators. There are seven indicators has loading values which is less than 0.50 . The seven indicators are PBAs2, PBAs4, PBKop2, PBKop3, PBOb2, PBOb3, and PBOb5. All seven indicators are deleted from the instruments.

Table I: Loadings Values

\begin{tabular}{|c|c|c|}
\hline Construct & Item & Loading Values \\
\hline \multirow{2}{*}{ Auditory } & GBD1 & 0.85 \\
\hline & GBD2 & 0.64 \\
\hline \multicolumn{3}{|c|}{$\begin{array}{l}\text { Published By: } \\
\text { Blue Eyes Intelligence Engineering } \\
\text { \& Sciences Publication } \\
\text { (C) Copyright: All rights reserved. }\end{array}$} \\
\hline
\end{tabular}




\begin{tabular}{|c|c|c|}
\hline & GBD3 & 0.81 \\
\hline \multirow{3}{*}{ Phrase } & GBF1 & 0.89 \\
\hline & GBF2 & 0.89 \\
\hline & GBF3 & 0.59 \\
\hline \multirow{3}{*}{ Kinesthetic } & GBG1 & 0.79 \\
\hline & GBG2 & 0.82 \\
\hline & GBG3 & 0.76 \\
\hline \multirow{3}{*}{ Individual } & GBI1 & 0.85 \\
\hline & GBI2 & 0.86 \\
\hline & GBI3 & 0.81 \\
\hline \multirow{3}{*}{ Group } & GBK1 & 0.78 \\
\hline & GBK2 & 0.82 \\
\hline & GBK3 & 0.81 \\
\hline \multirow{3}{*}{ Visual } & GBL1 & 0.88 \\
\hline & GBL2 & 0.74 \\
\hline & GBL3 & 0.84 \\
\hline \multirow{3}{*}{ Tactile } & GBP1 & 0.81 \\
\hline & GBP2 & 0.74 \\
\hline & GBP3 & 0.83 \\
\hline \multirow{5}{*}{ Active } & PBAk1 & 0.55 \\
\hline & PBAk2 & 0.55 \\
\hline & PBAk3 & 0.68 \\
\hline & PBAk4 & 0.62 \\
\hline & PBAk5 & 0.60 \\
\hline \multirow{4}{*}{ Authentic } & PBAs1 & 0.88 \\
\hline & PBAs2 & -0.05 \\
\hline & PBAs3 & 0.80 \\
\hline & PBAs4 & -0.19 \\
\hline \multirow{3}{*}{$\begin{array}{c}\text { Constructiv } \\
\mathrm{e}\end{array}$} & PBKon1 & 0.74 \\
\hline & PBKon2 & 0.60 \\
\hline & PBKon3 & 0.55 \\
\hline \multirow{4}{*}{ Cooperative } & PBKop1 & 0.70 \\
\hline & PBKop2 & 0.41 \\
\hline & PBКор3 & 0.15 \\
\hline & PBКор4 & 0.69 \\
\hline \multirow{6}{*}{ Objective } & PBOb1 & 0.62 \\
\hline & PBOb2 & 0.44 \\
\hline & PBOb3 & 0.26 \\
\hline & PBOb4 & 0.60 \\
\hline & PBOb5 & 0.46 \\
\hline & PBOb6 & 0.51 \\
\hline
\end{tabular}

\section{B. Construct Validity}

Construct validity refers to how far the result obtained specifically measures the intended construct [22]. Construct validity can be assessed using two types of validity: convergent and discriminant validity. The first requirement is that the values appear for each loading and cross loading, to verify whether there are problems with any particular items representing the construct. Table 1 represents respective values of loadings and cross loadings after deletion of seven item has been made based on the loading values shown in Table 1. Cutoff value used in this study for loadings to be significant was at 0.50 [21]. Therefore, any items which had a loading higher than 0.50 on two or more factors were assumed to be having significant cross loadings. In Table II, all the items measuring the construct assigned with highly loaded values, loaded lower on the other constructs. This shows that the construct validity of the item is supported.

Table II: Construct validity

\begin{tabular}{|c|c|c|c|c|c|c|c|c|c|c|c|c|}
\hline & $\begin{array}{c}\text { A } \\
\text { ud }\end{array}$ & $\begin{array}{l}\text { Ph } \\
\text { r. }\end{array}$ & $\begin{array}{l}\text { Ki } \\
\mathbf{n} .\end{array}$ & $\begin{array}{l}\text { In } \\
\text { d. }\end{array}$ & $\begin{array}{c}\text { Gr } \\
\text { o. }\end{array}$ & $\begin{array}{l}\text { Vi } \\
\text { s. }\end{array}$ & $\begin{array}{l}\text { Ta } \\
\text { c. }\end{array}$ & $\begin{array}{l}\text { A } \\
\text { ct. }\end{array}$ & $\begin{array}{l}\text { A } \\
\text { ut. }\end{array}$ & $\begin{array}{c}\text { C } \\
\text { on }\end{array}$ & $\begin{array}{c}\text { C } \\
\text { oo }\end{array}$ & $\begin{array}{c}\text { O } \\
\text { bj. }\end{array}$ \\
\hline GBD1 & $\begin{array}{r}0 . \\
85\end{array}$ & $\begin{array}{c}0 . \\
38\end{array}$ & $\begin{array}{r}0 . \\
34\end{array}$ & $\begin{array}{c}0 . \\
33\end{array}$ & $\begin{array}{c}0 . \\
38\end{array}$ & $\begin{array}{c}0 . \\
56\end{array}$ & $\begin{array}{c}0 . \\
48\end{array}$ & $\begin{array}{c}0 . \\
25\end{array}$ & $\begin{array}{c}0 . \\
08\end{array}$ & $\begin{array}{c}0 . \\
27\end{array}$ & $\begin{array}{c}0 . \\
01\end{array}$ & $\begin{array}{c}0 . \\
11\end{array}$ \\
\hline GBD2 & $\begin{array}{c}0 . \\
64\end{array}$ & $\begin{array}{c}0 . \\
33\end{array}$ & $\begin{array}{r}0 . \\
38 \\
\end{array}$ & $\begin{array}{c}0 . \\
06\end{array}$ & $\begin{array}{c}0 . \\
45\end{array}$ & $\begin{array}{l}0 . \\
51\end{array}$ & $\begin{array}{l}0 . \\
50\end{array}$ & $\begin{array}{l}0 . \\
17\end{array}$ & $\begin{array}{c}-0 . \\
03\end{array}$ & $\begin{array}{c}0 . \\
24\end{array}$ & $\begin{array}{c}0 . \\
20\end{array}$ & $\begin{array}{l}-0 . \\
11\end{array}$ \\
\hline GBD3 & $\begin{array}{c}\mathbf{0} \\
\mathbf{8 1}\end{array}$ & $\begin{array}{c}0 . \\
39\end{array}$ & $\begin{array}{c}0 . \\
33\end{array}$ & $\begin{array}{c}0 . \\
26\end{array}$ & $\begin{array}{c}0 . \\
44\end{array}$ & $\begin{array}{l}0 . \\
56\end{array}$ & $\begin{array}{c}0 . \\
50\end{array}$ & $\begin{array}{c}0 . \\
24\end{array}$ & $\begin{array}{c}0 . \\
20\end{array}$ & $\begin{array}{c}0 . \\
26\end{array}$ & $\begin{array}{c}0 . \\
16\end{array}$ & $\begin{array}{l}0 . \\
24\end{array}$ \\
\hline GBF1 & $\begin{array}{c}0 . \\
43\end{array}$ & $\begin{array}{c}0 . \\
89\end{array}$ & $\begin{array}{c}0 . \\
20\end{array}$ & $\begin{array}{c}0 . \\
40\end{array}$ & $\begin{array}{c}0 . \\
37\end{array}$ & $\begin{array}{l}0 . \\
48\end{array}$ & $\begin{array}{l}0 . \\
27\end{array}$ & $\begin{array}{c}0 . \\
18\end{array}$ & $\begin{array}{c}0 . \\
02\end{array}$ & $\begin{array}{c}0 . \\
18\end{array}$ & $\begin{array}{c}0 . \\
02\end{array}$ & $\begin{array}{l}0 . \\
16\end{array}$ \\
\hline GBF2 & $\begin{array}{c}0 . \\
48\end{array}$ & $\begin{array}{c}0 . \\
89\end{array}$ & $\begin{array}{c}0 . \\
39\end{array}$ & $\begin{array}{c}0 . \\
43\end{array}$ & $\begin{array}{c}0 . \\
41\end{array}$ & $\begin{array}{l}0 . \\
48\end{array}$ & $\begin{array}{c}0 . \\
45\end{array}$ & $\begin{array}{c}0 . \\
12\end{array}$ & $\begin{array}{c}0 . \\
16\end{array}$ & $\begin{array}{c}0 . \\
12\end{array}$ & $\begin{array}{c}0 . \\
00\end{array}$ & $\begin{array}{c}0 . \\
16\end{array}$ \\
\hline GBF3 & $\begin{array}{c}0 . \\
15\end{array}$ & $\begin{array}{c}0 . \\
59\end{array}$ & $\begin{array}{c}0 . \\
08\end{array}$ & $\begin{array}{c}0 . \\
38\end{array}$ & $\begin{array}{c}0 . \\
22\end{array}$ & $\begin{array}{l}0 . \\
23\end{array}$ & $\begin{array}{c}0 . \\
03\end{array}$ & $\begin{array}{c}0 . \\
14\end{array}$ & $\begin{array}{c}0 . \\
00\end{array}$ & $\begin{array}{c}0 . \\
16\end{array}$ & $\begin{array}{c}-0 . \\
04\end{array}$ & $\begin{array}{l}-0 . \\
24\end{array}$ \\
\hline $\begin{array}{c}\text { GBG } \\
1\end{array}$ & $\begin{array}{c}0 \\
40 \\
\end{array}$ & $\begin{array}{c}0 . \\
33 \\
\end{array}$ & $\begin{array}{c}0 . \\
79 \\
\end{array}$ & $\begin{array}{c}0 . \\
15 \\
\end{array}$ & $\begin{array}{c}0 . \\
49 \\
\end{array}$ & $\begin{array}{l}0 . \\
57\end{array}$ & $\begin{array}{c}0 . \\
39 \\
\end{array}$ & $\begin{array}{l}0 . \\
17\end{array}$ & $\begin{array}{c}0 . \\
15 \\
\end{array}$ & $\begin{array}{c}0 . \\
25 \\
\end{array}$ & $\begin{array}{l}-0 . \\
06 \\
\end{array}$ & $\begin{array}{c}0 . \\
12\end{array}$ \\
\hline $\begin{array}{c}\text { GBG } \\
2\end{array}$ & $\begin{array}{c}0 . \\
31 \\
\end{array}$ & $\begin{array}{c}0 \\
20 \\
\end{array}$ & $\begin{array}{r}\mathbf{0} \\
\mathbf{8 2} \\
\end{array}$ & $\begin{array}{c}0 . \\
30 \\
\end{array}$ & $\begin{array}{l}0 . \\
41 \\
\end{array}$ & $\begin{array}{l}0 . \\
47 \\
\end{array}$ & $\begin{array}{c}0 . \\
46 \\
\end{array}$ & $\begin{array}{l}0 \\
28 \\
\end{array}$ & $\begin{array}{c}0 . \\
16 \\
\end{array}$ & $\begin{array}{c}0 . \\
31 \\
\end{array}$ & $\begin{array}{c}0 . \\
06 \\
\end{array}$ & $\begin{array}{l}0 . \\
17 \\
\end{array}$ \\
\hline $\begin{array}{c}\text { GBG } \\
3\end{array}$ & $\begin{array}{c}0 . \\
35\end{array}$ & $\begin{array}{l}0 . \\
19\end{array}$ & $\begin{array}{c}0 . \\
76\end{array}$ & $\begin{array}{c}0 . \\
13\end{array}$ & $\begin{array}{l}0 . \\
41\end{array}$ & $\begin{array}{l}0 . \\
57\end{array}$ & $\begin{array}{l}0 . \\
48\end{array}$ & $\begin{array}{c}0 . \\
11\end{array}$ & $\begin{array}{c}0 . \\
09\end{array}$ & $\begin{array}{c}0 . \\
33\end{array}$ & $\begin{array}{c}0 . \\
14\end{array}$ & $\begin{array}{c}0 . \\
03\end{array}$ \\
\hline GBI1 & $\begin{array}{c}0 . \\
36 \\
\end{array}$ & $\begin{array}{l}0 . \\
45 \\
\end{array}$ & $\begin{array}{l}0 . \\
26 \\
\end{array}$ & $\begin{array}{c}\mathbf{0 .} \\
85\end{array}$ & $\begin{array}{l}0 . \\
19 \\
\end{array}$ & $\begin{array}{c}0 . \\
34 \\
\end{array}$ & $\begin{array}{l}0 . \\
32\end{array}$ & $\begin{array}{l}0 . \\
04\end{array}$ & $\begin{array}{l}0 . \\
20\end{array}$ & $\begin{array}{l}0 . \\
27\end{array}$ & $\begin{array}{l}-0 . \\
05 \\
\end{array}$ & $\begin{array}{l}0 . \\
07\end{array}$ \\
\hline GBI2 & $\begin{array}{c}0 . \\
16 \\
\end{array}$ & $\begin{array}{c}0 . \\
36 \\
\end{array}$ & $\begin{array}{c}0 . \\
07 \\
\end{array}$ & $\begin{array}{c}0 . \\
86 \\
\end{array}$ & $\begin{array}{c}0 . \\
22 \\
\end{array}$ & $\begin{array}{c}0 . \\
10 \\
\end{array}$ & $\begin{array}{c}0 . \\
05 \\
\end{array}$ & $\begin{array}{c}0 . \\
21 \\
\end{array}$ & $\begin{array}{l}-0 . \\
01 \\
\end{array}$ & $\begin{array}{c}0 . \\
28 \\
\end{array}$ & $\begin{array}{c}0 . \\
14 \\
\end{array}$ & $\begin{array}{c}0 . \\
08 \\
\end{array}$ \\
\hline GBI3 & $\begin{array}{c}0 . \\
15\end{array}$ & $\begin{array}{l}0 . \\
41\end{array}$ & $\begin{array}{c}0 . \\
23\end{array}$ & $\begin{array}{c}0 . \\
81\end{array}$ & $\begin{array}{c}0 . \\
26\end{array}$ & $\begin{array}{c}0 . \\
21\end{array}$ & $\begin{array}{c}0 . \\
09\end{array}$ & $\begin{array}{c}0 . \\
24\end{array}$ & $\begin{array}{c}0 . \\
05\end{array}$ & $\begin{array}{c}0 . \\
26\end{array}$ & $\begin{array}{c}0 . \\
17\end{array}$ & $\begin{array}{c}0 . \\
10\end{array}$ \\
\hline $\begin{array}{c}\text { GBK } \\
1 \\
\end{array}$ & $\begin{array}{l}0 . \\
48 \\
\end{array}$ & $\begin{array}{l}0 . \\
27 \\
\end{array}$ & $\begin{array}{r}0 . \\
35 \\
\end{array}$ & $\begin{array}{l}0 . \\
13 \\
\end{array}$ & $\begin{array}{l}0 . \\
78 \\
\end{array}$ & $\begin{array}{l}0 . \\
45 \\
\end{array}$ & $\begin{array}{c}0 . \\
39 \\
\end{array}$ & $\begin{array}{l}0 . \\
32 \\
\end{array}$ & $\begin{array}{c}0 . \\
10 \\
\end{array}$ & $\begin{array}{c}0 . \\
28 \\
\end{array}$ & $\begin{array}{l}0 . \\
17 \\
\end{array}$ & $\begin{array}{c}0 . \\
17 \\
\end{array}$ \\
\hline $\begin{array}{c}\text { GBK } \\
2 \\
\end{array}$ & $\begin{array}{c}0 \\
40 \\
\end{array}$ & $\begin{array}{c}0 . \\
28 \\
\end{array}$ & $\begin{array}{c}0 . \\
53 \\
\end{array}$ & $\begin{array}{c}0 . \\
25 \\
\end{array}$ & $\begin{array}{c}0 . \\
82 \\
\end{array}$ & $\begin{array}{l}0 . \\
40\end{array}$ & $\begin{array}{c}0 . \\
31\end{array}$ & $\begin{array}{l}0 . \\
41\end{array}$ & $\begin{array}{c}0 . \\
18\end{array}$ & $\begin{array}{l}0 . \\
42\end{array}$ & $\begin{array}{c}0 . \\
12\end{array}$ & $\begin{array}{c}0 . \\
12\end{array}$ \\
\hline $\begin{array}{c}\text { GBK } \\
3\end{array}$ & $\begin{array}{c}0 . \\
45\end{array}$ & $\begin{array}{l}0 . \\
47\end{array}$ & $\begin{array}{c}0 . \\
44\end{array}$ & $\begin{array}{c}0 . \\
23\end{array}$ & $\begin{array}{c}0 . \\
81\end{array}$ & $\begin{array}{l}0 . \\
45\end{array}$ & $\begin{array}{l}0 . \\
41\end{array}$ & $\begin{array}{c}0 . \\
31\end{array}$ & $\begin{array}{c}0 . \\
15\end{array}$ & $\begin{array}{c}0 . \\
33\end{array}$ & $\begin{array}{c}0 . \\
17\end{array}$ & $\begin{array}{c}0 . \\
12\end{array}$ \\
\hline GBL1 & $\begin{array}{l}0 . \\
57\end{array}$ & $\begin{array}{l}0 . \\
51\end{array}$ & $\begin{array}{l}0 . \\
66 \\
\end{array}$ & $\begin{array}{l}0 . \\
24 \\
\end{array}$ & $\begin{array}{l}0 . \\
49 \\
\end{array}$ & $\begin{array}{c}0 . \\
88\end{array}$ & $\begin{array}{l}0 . \\
54\end{array}$ & $\begin{array}{l}0 . \\
17 \\
\end{array}$ & $\begin{array}{l}0 . \\
17 \\
\end{array}$ & $\begin{array}{c}0 . \\
25 \\
\end{array}$ & $\begin{array}{l}0 . \\
02\end{array}$ & $\begin{array}{l}-0 . \\
03 \\
\end{array}$ \\
\hline GBL2 & $\begin{array}{l}0 . \\
68 \\
\end{array}$ & $\begin{array}{c}0 . \\
43 \\
\end{array}$ & $\begin{array}{c}0 . \\
39 \\
\end{array}$ & $\begin{array}{c}0 . \\
20 \\
\end{array}$ & $\begin{array}{l}0 . \\
40 \\
\end{array}$ & $\begin{array}{c}0 . \\
74 \\
\end{array}$ & $\begin{array}{l}0 . \\
43 \\
\end{array}$ & $\begin{array}{c}0 . \\
17 \\
\end{array}$ & $\begin{array}{c}0 . \\
09 \\
\end{array}$ & $\begin{array}{c}0 . \\
35 \\
\end{array}$ & $\begin{array}{c}0 . \\
00 \\
\end{array}$ & $\begin{array}{l}-0 . \\
04 \\
\end{array}$ \\
\hline GBL3 & $\begin{array}{l}0 . \\
50\end{array}$ & $\begin{array}{l}0 . \\
34\end{array}$ & $\begin{array}{l}0 . \\
61\end{array}$ & $\begin{array}{c}0 . \\
26\end{array}$ & $\begin{array}{l}0 . \\
44\end{array}$ & $\begin{array}{c}0 . \\
84\end{array}$ & $\begin{array}{l}0 . \\
57\end{array}$ & 30 & $\begin{array}{c}0 . \\
28\end{array}$ & $\begin{array}{c}0 . \\
37\end{array}$ & $\begin{array}{c}0 . \\
20\end{array}$ & $\begin{array}{c}0 . \\
09\end{array}$ \\
\hline GBP1 & $\begin{array}{l}0 . \\
54 \\
\end{array}$ & $\begin{array}{c}0 . \\
32 \\
\end{array}$ & $\begin{array}{l}0 . \\
37 \\
\end{array}$ & $\begin{array}{c}0 . \\
16 \\
\end{array}$ & $\begin{array}{l}0 . \\
37 \\
\end{array}$ & $\begin{array}{l}0 . \\
49 \\
\end{array}$ & $\begin{array}{l}0 . \\
81\end{array}$ & $\begin{array}{l}0 . \\
16 \\
\end{array}$ & $\begin{array}{l}0 . \\
22 \\
\end{array}$ & $\begin{array}{c}0 . \\
11 \\
\end{array}$ & $\begin{array}{l}0 . \\
18 \\
\end{array}$ & $\begin{array}{r}-0 . \\
07 \\
\end{array}$ \\
\hline GBP2 & $\begin{array}{c}0 . \\
41 \\
\end{array}$ & $\begin{array}{c}0 . \\
08 \\
\end{array}$ & $\begin{array}{c}0 . \\
50 \\
\end{array}$ & $\begin{array}{c}0 . \\
19 \\
\end{array}$ & $\begin{array}{c}0 . \\
29 \\
\end{array}$ & $\begin{array}{c}0 . \\
40\end{array}$ & $\begin{array}{c}0 . \\
74 \\
\end{array}$ & $\begin{array}{c}0 . \\
22 \\
\end{array}$ & $\begin{array}{c}0 . \\
29 \\
\end{array}$ & $\begin{array}{c}0 . \\
31 \\
\end{array}$ & $\begin{array}{c}0 . \\
14 \\
\end{array}$ & $\begin{array}{c}0 . \\
09 \\
\end{array}$ \\
\hline GBP3 & $\begin{array}{l}0 . \\
55\end{array}$ & $\begin{array}{l}0 . \\
42\end{array}$ & $\begin{array}{c}0 . \\
48\end{array}$ & $\begin{array}{c}0 . \\
16\end{array}$ & $\begin{array}{c}0 . \\
43\end{array}$ & $\begin{array}{c}0 . \\
59\end{array}$ & $\begin{array}{r}0 . \\
83\end{array}$ & $\begin{array}{c}0 . \\
24\end{array}$ & $\begin{array}{c}0 . \\
22\end{array}$ & $\begin{array}{c}0 . \\
21\end{array}$ & $\begin{array}{c}0 . \\
17\end{array}$ & $\begin{array}{c}0 . \\
18\end{array}$ \\
\hline $\begin{array}{c}\text { PBAk } \\
1\end{array}$ & $\begin{array}{l}0 . \\
21 \\
\end{array}$ & $\begin{array}{c}0 . \\
02\end{array}$ & $\begin{array}{l}0 . \\
17 \\
\end{array}$ & $\begin{array}{c}0 . \\
11 \\
\end{array}$ & $\begin{array}{l}0 . \\
25 \\
\end{array}$ & $\begin{array}{c}0 . \\
16 \\
\end{array}$ & $\begin{array}{l}0 . \\
13 \\
\end{array}$ & $\begin{array}{c}0 . \\
55 \\
\end{array}$ & $\begin{array}{l}0 . \\
01\end{array}$ & $\begin{array}{c}0 . \\
39 \\
\end{array}$ & $\begin{array}{l}0 . \\
17\end{array}$ & $\begin{array}{l}0 . \\
30 \\
\end{array}$ \\
\hline $\begin{array}{c}\text { PBAk } \\
2\end{array}$ & $\begin{array}{c}0 . \\
09\end{array}$ & $\begin{array}{c}0 . \\
11\end{array}$ & $\begin{array}{c}0 . \\
14\end{array}$ & $\begin{array}{c}0 . \\
21\end{array}$ & $\begin{array}{l}0 . \\
24\end{array}$ & $\begin{array}{c}0 . \\
14\end{array}$ & $\begin{array}{c}0 . \\
05\end{array}$ & $\begin{array}{c}0 . \\
55\end{array}$ & $\begin{array}{c}0 . \\
29\end{array}$ & $\begin{array}{c}0 . \\
30\end{array}$ & $\begin{array}{c}0 . \\
36\end{array}$ & $\begin{array}{l}0 . \\
21\end{array}$ \\
\hline $\begin{array}{c}\text { PBAk } \\
3\end{array}$ & $\begin{array}{c}0 . \\
10\end{array}$ & $\begin{array}{c}0 . \\
11\end{array}$ & $\begin{array}{c}0 . \\
05\end{array}$ & $\begin{array}{c}0 . \\
11\end{array}$ & $\begin{array}{c}0 . \\
21\end{array}$ & $\begin{array}{c}0 . \\
12\end{array}$ & $\begin{array}{c}0 . \\
14\end{array}$ & $\begin{array}{c}0 . \\
68\end{array}$ & $\begin{array}{c}0 . \\
40\end{array}$ & $\begin{array}{c}0 . \\
26\end{array}$ & $\begin{array}{c}0 . \\
36\end{array}$ & $\begin{array}{c}0 . \\
29\end{array}$ \\
\hline $\begin{array}{c}\text { PBAk } \\
4 \\
\end{array}$ & $\begin{array}{l}0 . \\
19 \\
\end{array}$ & $\begin{array}{c}0 . \\
13 \\
\end{array}$ & $\begin{array}{l}0 . \\
19 \\
\end{array}$ & $\begin{array}{c}0 . \\
07\end{array}$ & $\begin{array}{c}0 . \\
35 \\
\end{array}$ & $\begin{array}{c}0 . \\
15 \\
\end{array}$ & $\begin{array}{l}0 . \\
21 \\
\end{array}$ & $\begin{array}{l}0 . \\
63 \\
\end{array}$ & $\begin{array}{l}0 . \\
26 \\
\end{array}$ & $\begin{array}{c}0 . \\
26 \\
\end{array}$ & $\begin{array}{l}0 . \\
18 \\
\end{array}$ & $\begin{array}{l}0 . \\
41 \\
\end{array}$ \\
\hline $\begin{array}{c}\text { PBAk } \\
5\end{array}$ & $\begin{array}{c}0 . \\
29 \\
\end{array}$ & $\begin{array}{c}0 . \\
14\end{array}$ & $\begin{array}{c}0 . \\
18 \\
\end{array}$ & $\begin{array}{c}0 . \\
02 \\
\end{array}$ & $\begin{array}{c}0 . \\
26\end{array}$ & $\begin{array}{l}0 . \\
21\end{array}$ & $\begin{array}{l}0 . \\
25\end{array}$ & $\begin{array}{l}0 . \\
60\end{array}$ & $\begin{array}{c}0 . \\
27\end{array}$ & $\begin{array}{l}0 . \\
25\end{array}$ & $\begin{array}{c}0 . \\
07\end{array}$ & $\begin{array}{l}0 . \\
25\end{array}$ \\
\hline $\begin{array}{c}\text { PBAs } \\
1\end{array}$ & $\begin{array}{c}0 . \\
09\end{array}$ & $\begin{array}{c}0 . \\
17\end{array}$ & $\begin{array}{c}0 . \\
14\end{array}$ & $\begin{array}{l}0 . \\
21\end{array}$ & $\begin{array}{c}0 . \\
16\end{array}$ & $\begin{array}{l}0 . \\
15\end{array}$ & $\begin{array}{c}0 . \\
21\end{array}$ & $\begin{array}{l}0 . \\
41\end{array}$ & $\begin{array}{c}\mathbf{0 .} \\
\mathbf{8 8}\end{array}$ & $\begin{array}{c}0 . \\
33\end{array}$ & $\begin{array}{c}0 . \\
28\end{array}$ & $\begin{array}{c}0 . \\
30\end{array}$ \\
\hline $\begin{array}{c}\text { PBAs } \\
3 \\
\end{array}$ & $\begin{array}{c}0 . \\
11 \\
\end{array}$ & $\begin{array}{l}-0 . \\
03 \\
\end{array}$ & $\begin{array}{l}0 . \\
15 \\
\end{array}$ & $\begin{array}{l}-0 . \\
05 \\
\end{array}$ & $\begin{array}{c}0 . \\
14 \\
\end{array}$ & $\begin{array}{l}0 . \\
23 \\
\end{array}$ & $\begin{array}{l}0 . \\
31 \\
\end{array}$ & $\begin{array}{l}0 . \\
31 \\
\end{array}$ & $\begin{array}{l}0 . \\
82 \\
\end{array}$ & $\begin{array}{c}0 . \\
31 \\
\end{array}$ & $\begin{array}{c}0 . \\
10 \\
\end{array}$ & $\begin{array}{l}0 . \\
20\end{array}$ \\
\hline $\begin{array}{c}\text { PBKo } \\
\text { n1 }\end{array}$ & $\begin{array}{l}0 . \\
22 \\
\end{array}$ & $\begin{array}{l}0 . \\
23 \\
\end{array}$ & $\begin{array}{c}0 . \\
30 \\
\end{array}$ & $\begin{array}{c}0 . \\
35 \\
\end{array}$ & $\begin{array}{l}0 . \\
28 \\
\end{array}$ & $\begin{array}{c}0 . \\
34 \\
\end{array}$ & $\begin{array}{l}0 . \\
25 \\
\end{array}$ & $\begin{array}{c}0 . \\
31 \\
\end{array}$ & $\begin{array}{l}0 . \\
29 \\
\end{array}$ & $\begin{array}{l}0 . \\
75 \\
\end{array}$ & $\begin{array}{l}0 . \\
15 \\
\end{array}$ & $\begin{array}{r}0 . \\
34 \\
\end{array}$ \\
\hline $\begin{array}{c}\text { PBKo } \\
\text { n2 }\end{array}$ & $\begin{array}{c}0 . \\
21\end{array}$ & $\begin{array}{c}0 . \\
11\end{array}$ & $\begin{array}{c}0 . \\
20\end{array}$ & $\begin{array}{c}0 . \\
19\end{array}$ & $\begin{array}{c}0 . \\
29\end{array}$ & $\begin{array}{c}0 . \\
21\end{array}$ & $\begin{array}{l}0 . \\
17\end{array}$ & $\begin{array}{c}0 . \\
22\end{array}$ & $\begin{array}{c}0 . \\
24\end{array}$ & $\begin{array}{c}0 . \\
61\end{array}$ & $\begin{array}{c}-0 . \\
08\end{array}$ & $\begin{array}{r}0 . \\
32\end{array}$ \\
\hline $\begin{array}{c}\text { PBKo } \\
\text { n3 }\end{array}$ & $\begin{array}{l}0 . \\
20 \\
\end{array}$ & $\begin{array}{l}-0 . \\
02 \\
\end{array}$ & $\begin{array}{c}0 . \\
20 \\
\end{array}$ & $\begin{array}{c}0 . \\
03\end{array}$ & $\begin{array}{l}0 . \\
26 \\
\end{array}$ & $\begin{array}{l}0 . \\
17\end{array}$ & $\begin{array}{c}0 . \\
05 \\
\end{array}$ & $\begin{array}{l}0 . \\
38 \\
\end{array}$ & $\begin{array}{l}0 . \\
17 \\
\end{array}$ & $\begin{array}{c}0 . \\
53 \\
\end{array}$ & $\begin{array}{l}0 . \\
22 \\
\end{array}$ & $\begin{array}{l}-0 . \\
01\end{array}$ \\
\hline $\begin{array}{c}\text { PBKo } \\
\text { p1 }\end{array}$ & $\begin{array}{c}0 . \\
13\end{array}$ & $\begin{array}{c}-0 . \\
10\end{array}$ & $\begin{array}{c}0 . \\
04\end{array}$ & $\begin{array}{l}-0 . \\
03\end{array}$ & $\begin{array}{c}0 . \\
11\end{array}$ & $\begin{array}{l}-0 . \\
04\end{array}$ & $\begin{array}{c}0 . \\
03\end{array}$ & $\begin{array}{c}0 . \\
28\end{array}$ & $\begin{array}{c}0 . \\
14\end{array}$ & $\begin{array}{c}0 . \\
09\end{array}$ & $\begin{array}{c}0 . \\
72\end{array}$ & $\begin{array}{r}0 . \\
19\end{array}$ \\
\hline $\begin{array}{c}\text { PBKo } \\
\text { p4 }\end{array}$ & $\begin{array}{c}0 . \\
11 \\
\end{array}$ & $\begin{array}{c}0 . \\
08 \\
\end{array}$ & $\begin{array}{c}0 . \\
05 \\
\end{array}$ & $\begin{array}{c}0 . \\
14 \\
\end{array}$ & $\begin{array}{l}0 . \\
17 \\
\end{array}$ & $\begin{array}{c}0 . \\
16 \\
\end{array}$ & $\begin{array}{l}0 . \\
27 \\
\end{array}$ & $\begin{array}{c}0 . \\
31 \\
\end{array}$ & $\begin{array}{c}0 . \\
20 \\
\end{array}$ & $\begin{array}{c}0 . \\
15 \\
\end{array}$ & $\begin{array}{c}\mathbf{0 .} \\
\mathbf{8 0} \\
\end{array}$ & $\begin{array}{c}0 . \\
16 \\
\end{array}$ \\
\hline $\begin{array}{c}\text { PBOb } \\
1\end{array}$ & $\begin{array}{l}-0 . \\
05 \\
\end{array}$ & $\begin{array}{c}0 . \\
04\end{array}$ & $\begin{array}{r}-0 . \\
02 \\
\end{array}$ & $\begin{array}{l}-0 . \\
11 \\
\end{array}$ & $\begin{array}{l}-0 . \\
04\end{array}$ & $\begin{array}{l}-0 . \\
13 \\
\end{array}$ & $\begin{array}{l}-0 . \\
03 \\
\end{array}$ & $\begin{array}{l}0 . \\
34 \\
\end{array}$ & $\begin{array}{l}0 . \\
18 \\
\end{array}$ & $\begin{array}{c}0 . \\
16 \\
\end{array}$ & $\begin{array}{l}0 . \\
25 \\
\end{array}$ & $\begin{array}{r}0 . \\
72\end{array}$ \\
\hline $\begin{array}{c}\mathrm{PBOb} \\
4\end{array}$ & $\begin{array}{c}0 . \\
16\end{array}$ & $\begin{array}{c}0 . \\
03\end{array}$ & $\begin{array}{l}0 . \\
22\end{array}$ & $\begin{array}{c}0 . \\
15\end{array}$ & $\begin{array}{c}0 . \\
18\end{array}$ & $\begin{array}{c}0 . \\
04\end{array}$ & $\begin{array}{c}0 . \\
24\end{array}$ & $\begin{array}{l}0 . \\
37\end{array}$ & $\begin{array}{c}0 . \\
36\end{array}$ & $\begin{array}{c}0 . \\
31\end{array}$ & $\begin{array}{c}0 . \\
09\end{array}$ & $\begin{array}{r}0 . \\
71\end{array}$ \\
\hline $\begin{array}{c}\text { PBOb } \\
6\end{array}$ & $\begin{array}{c}0 . \\
12\end{array}$ & $\begin{array}{c}0 . \\
15\end{array}$ & $\begin{array}{c}0 . \\
07\end{array}$ & $\begin{array}{c}0 . \\
16\end{array}$ & $\begin{array}{c}0 . \\
21\end{array}$ & $\begin{array}{c}0 . \\
11\end{array}$ & $\begin{array}{c}-0 . \\
08\end{array}$ & $\begin{array}{c}0 . \\
29\end{array}$ & $\begin{array}{c}0 . \\
03\end{array}$ & $\begin{array}{c}0 . \\
27\end{array}$ & $\begin{array}{c}0 . \\
14\end{array}$ & $\begin{array}{r}0 . \\
63\end{array}$ \\
\hline
\end{tabular}

Bold values: loadings for items, which fulfill the recommended value $(>0.50)[21]$

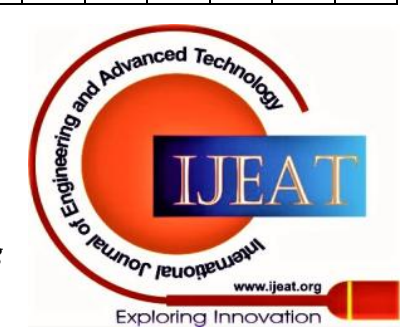




\section{The Impact of Orang Asli Students' Learning Styles on Their Achievement of Meaningful Learning}

\section{Convergent Validity}

Second, convergent validity was tested. This validity refers to the degree to which multiple items measure the same concepts are in line. For this purpose, three importance criteria needed to be taken into consideration. They were (i) factor loadings, (ii) composite reliability (CR), and (iii) average variance extracted (AVE) [21]. Table III shows the values of these three important criteria after deletion four item which is PBAk1, PBAk2, PBAk4, and PBKon3 has been made based on the values of AVE which is less than 0.50. Deleting the item leads to an increase in the AVE values. Loadings for all items are higher than the recommended values of 0.50 [21], while the composite reliability values ranged from 0.73 to 0.88 that exceeded the recommended value of 0.70 [21]. The last criteria are the values of average variance extracted (AVE). AVE should be higher than 0.50 to justify the construct [23]. The AVE range is between 0.50 and 0.73 .

Table III: Convergent validity

\begin{tabular}{|c|c|c|c|c|c|}
\hline Component & Construct & $\begin{array}{l}\text { Measurement } \\
\text { Item }\end{array}$ & Loading & $\mathbf{C R}^{\mathrm{a}}$ & $\underset{b}{\operatorname{AVE}}$ \\
\hline \multirow{21}{*}{$\begin{array}{l}\text { Learning } \\
\text { Styles }\end{array}$} & \multirow[t]{3}{*}{ Group } & GBK1 & 0.78 & 0.84 & 0.64 \\
\hline & & GBK2 & 0.82 & & \\
\hline & & GBK3 & 0.81 & & \\
\hline & \multirow[t]{3}{*}{ Individual } & GBI1 & 0.85 & 088 & 070 \\
\hline & & GBI2 & 0.86 & & \\
\hline & & GBI3 & 0.81 & & \\
\hline & \multirow[t]{3}{*}{ Kinesthetic } & GBG1 & 0.79 & 083 & 0,63 \\
\hline & & GBG2 & 0.82 & & \\
\hline & & GBG3 & 0.77 & & \\
\hline & \multirow[t]{3}{*}{ Auditory } & GBD1 & 0.85 & 082 & 0.60 \\
\hline & & GBD2 & 0.64 & & \\
\hline & & GBD3 & 0.81 & & \\
\hline & \multirow[t]{3}{*}{ Phrase } & GBF1 & 0.89 & 0.84 & 0.65 \\
\hline & & GBF2 & 0.89 & & \\
\hline & & GBF3 & 0.60 & & \\
\hline & \multirow[t]{3}{*}{ Tactile } & GBP1 & 0.81 & 0.84 & 0.63 \\
\hline & & GBP2 & 0.74 & & \\
\hline & & GBP3 & 0.83 & & \\
\hline & \multirow[t]{3}{*}{ Visual } & GBL1 & 0.88 & 086 & 068 \\
\hline & & GBL2 & 0.74 & & \\
\hline & & GBL3 & 0.84 & & \\
\hline \multirow{11}{*}{$\begin{array}{l}\text { Meaningful } \\
\text { Learning }\end{array}$} & \multirow[t]{2}{*}{ Active } & PBAk3 & 0.87 & 079 & 065 \\
\hline & & PBAk5 & 0.74 & & \\
\hline & \multirow[t]{2}{*}{ Authentic } & PBAs1 & 0.89 & 0.84 & 0.73 \\
\hline & & PBAs3 & 0.81 & & \\
\hline & \multirow[t]{3}{*}{ Objective } & PBOb1 & 0.73 & 073 & 0.50 \\
\hline & & PBOb4 & 0.72 & & \\
\hline & & PBOk6 & 0.62 & & \\
\hline & \multirow[t]{2}{*}{ Constructive } & PBKon1 & 0.85 & 075 & 066 \\
\hline & & PBKon2 & 0.70 & & \\
\hline & \multirow[t]{2}{*}{ Cooperative } & PBKop1 & 0.70 & 0.73 & 0.58 \\
\hline & & PBKop4 & 0.82 & & \\
\hline
\end{tabular}

${ }^{\text {a }}$ Composite reliability $(\mathrm{CR})=$ (square of the summation of the factor loadings $) /\{$ (square of the summation of the factor loadings) + (square of the summation of the error variances) $\}[21]$

b Average variance extracted (AVE) $=$ (summation of the square of the factor
loadings) $/\{$ (summation of the square of the factor loadings $)+($ summation of the error
variances $)\}[23]$

\section{Discriminant Validity}

Third, the discriminant validity of the constructs was tested. The discriminant validity of the measures (the degree to which items differentiate among constructs or measure distinct concepts) was assessed by examining the correlations between the measures of potentially overlapping constructs [24]. Items should load more strongly on their own constructs in the model, and the average variance shared between each construct and its measures should be greater than the variance shared between the construct and other constructs [25]. Table IV shows that the squared correlations for each construct are less than the AVE for the indicators measuring that construct. This indicates adequate discriminant validity for the constructs

Table IV: Discriminant validity of construct

\begin{tabular}{|c|c|c|c|c|c|c|c|c|c|c|c|c|}
\hline & $\begin{array}{l}\text { A } \\
\text { ct. }\end{array}$ & $\begin{array}{l}\text { A } \\
\text { u } \\
\text { d. }\end{array}$ & $\begin{array}{l}\text { A } \\
\text { ut }\end{array}$ & $\begin{array}{c}\text { C } \\
\text { on }\end{array}$ & $\begin{array}{c}\text { C } \\
\text { oo }\end{array}$ & $\begin{array}{l}\text { G } \\
\text { ro }\end{array}$ & $\begin{array}{l}\text { In } \\
\text { d. }\end{array}$ & $\begin{array}{l}\mathbf{K i} \\
\mathbf{n} .\end{array}$ & $\begin{array}{c}\mathbf{O} \\
\text { bj }\end{array}$ & $\begin{array}{c}\mathbf{P} \\
\mathbf{h r}\end{array}$ & $\begin{array}{l}T \\
\text { ac }\end{array}$ & $\begin{array}{l}\text { Vi } \\
\text { s. }\end{array}$ \\
\hline Active & $\begin{array}{l}0 . \\
81\end{array}$ & & & & & & & & & & & \\
\hline Auditory & $\begin{array}{l}0 . \\
22\end{array}$ & $\begin{array}{l}\mathbf{0 .} \\
77\end{array}$ & & & & & & & & & & \\
\hline Authentic & $\begin{array}{l}0 . \\
42 \\
\end{array}$ & $\begin{array}{l}0 . \\
11\end{array}$ & $\begin{array}{l}0 . \\
85\end{array}$ & & & & & & & & & \\
\hline $\begin{array}{l}\text { Constructi } \\
\text { ve }\end{array}$ & $\begin{array}{l}0 . \\
21 \\
\end{array}$ & $\begin{array}{l}0 . \\
27 \\
\end{array}$ & $\begin{array}{l}0 . \\
34 \\
\end{array}$ & $\begin{array}{l}0 . \\
78 \\
\end{array}$ & & & & & & & & \\
\hline $\begin{array}{l}\text { Cooperati } \\
\text { ve }\end{array}$ & $\begin{array}{l}0 . \\
29\end{array}$ & $\begin{array}{l}0 . \\
15 \\
\end{array}$ & $\begin{array}{l}0 . \\
23\end{array}$ & $\begin{array}{l}0 . \\
07\end{array}$ & $\begin{array}{l}0 . \\
76 \\
\end{array}$ & & & & & & & \\
\hline Group & $\begin{array}{l}0 . \\
28\end{array}$ & $\begin{array}{l}0 . \\
55\end{array}$ & $\begin{array}{l}0 . \\
18 \\
\end{array}$ & $\begin{array}{l}0 . \\
36\end{array}$ & $\begin{array}{l}0 . \\
19 \\
\end{array}$ & $\begin{array}{l}\mathbf{0 .} \\
80\end{array}$ & & & & & & \\
\hline Individual & $\begin{array}{l}0 . \\
09\end{array}$ & $\begin{array}{l}0 . \\
29\end{array}$ & $\begin{array}{l}0 . \\
12 \\
\end{array}$ & $\begin{array}{l}0 . \\
36 \\
\end{array}$ & $\begin{array}{l}0 . \\
09 \\
\end{array}$ & $\begin{array}{l}0 . \\
26\end{array}$ & $\begin{array}{l}0 . \\
84\end{array}$ & & & & & \\
\hline Kinestheti & $\begin{array}{l}0 . \\
13\end{array}$ & $\begin{array}{l}0 . \\
45\end{array}$ & $\begin{array}{l}0 . \\
17\end{array}$ & $\begin{array}{l}0 . \\
33\end{array}$ & $\begin{array}{l}0 . \\
06\end{array}$ & $\begin{array}{l}0 . \\
55\end{array}$ & $\begin{array}{l}0 . \\
24\end{array}$ & $\begin{array}{l}0 . \\
79\end{array}$ & & & & \\
\hline Objective & $\begin{array}{l}0 . \\
34\end{array}$ & $\begin{array}{l}0 . \\
11\end{array}$ & $\begin{array}{l}0 . \\
30\end{array}$ & $\begin{array}{l}0 . \\
42\end{array}$ & $\begin{array}{l}0 . \\
23\end{array}$ & $\begin{array}{l}0 . \\
17\end{array}$ & $\begin{array}{l}0 . \\
10\end{array}$ & $\begin{array}{l}0 . \\
14\end{array}$ & $\begin{array}{l}0 . \\
69\end{array}$ & & & \\
\hline Phrase & $\begin{array}{l}0 . \\
15 \\
\end{array}$ & $\begin{array}{l}0 . \\
48 \\
\end{array}$ & $\begin{array}{l}0 . \\
09\end{array}$ & $\begin{array}{l}0 . \\
22\end{array}$ & $\begin{array}{l}0 . \\
00\end{array}$ & $\begin{array}{l}0 . \\
43\end{array}$ & $\begin{array}{l}0 . \\
50\end{array}$ & $\begin{array}{l}0 . \\
31\end{array}$ & $\begin{array}{l}0 . \\
09\end{array}$ & $\begin{array}{l}0 . \\
80\end{array}$ & & \\
\hline Tactile & $\begin{array}{l}0 . \\
23 \\
\end{array}$ & \begin{tabular}{|l|}
0. \\
64 \\
\end{tabular} & $\begin{array}{l}0 . \\
30 \\
\end{array}$ & $\begin{array}{l}0 . \\
28 \\
\end{array}$ & $\begin{array}{l}0 . \\
21 \\
\end{array}$ & $\begin{array}{ll}0 . \\
46 \\
\end{array}$ & $\begin{array}{l}0 . \\
21 \\
\end{array}$ & $\begin{array}{l}0 . \\
56 \\
\end{array}$ & \begin{tabular}{|l}
0. \\
09 \\
\end{tabular} & $\begin{array}{l}0 . \\
36 \\
\end{array}$ & $\begin{array}{l}0 . \\
80 \\
\end{array}$ & \\
\hline Visual & $\begin{array}{l}0 . \\
19 \\
\end{array}$ & $\begin{array}{l}0 . \\
70\end{array}$ & $\begin{array}{l}0 . \\
22\end{array}$ & $\begin{array}{l}0 . \\
36\end{array}$ & $\begin{array}{l}0 . \\
09\end{array}$ & $\begin{array}{l}0 . \\
54\end{array}$ & $\begin{array}{l}0 . \\
28 \\
\end{array}$ & $\begin{array}{l}0 . \\
68\end{array}$ & $\begin{array}{l}0 . \\
01\end{array}$ & $\begin{array}{l}0 . \\
52 \\
\end{array}$ & $\begin{array}{l}0 . \\
63 \\
\end{array}$ & $\begin{array}{l}0 . \\
82\end{array}$ \\
\hline
\end{tabular}

Bold values represent the AVE while the other entries represent the squared correlations [25]

\section{E. Hypothesis Testing}

Path analysis was conducted to test the research hypotheses. Figure 1 and Table 5 shows these results. Based on Figure 1, $R^{2}$ generated was 0.13 . This value suggests that $13 \%$ of the variance in meaningful learning can be explained by learning styles and that there was a positive relationship value of Beta equal to 0.36 which is higher than 0.20 , while the t-value equals to 3.07 ( $\mathrm{t}>1.645$ at $\mathrm{p}<0.05$ ) [24]. The other 12 hypothesis of this study were also supported. All 13 hypotheses complied with the criteria of hypothesis testing as the value of Beta was higher than 0.20 , and $t$-value exceeded 1.645 at $p$ less than 0.05 . Table V shows the summary of the hypotheses for the study.

Table V: Hypothesis testing

\begin{tabular}{|c|l|c|c|c|c|}
\hline Hypothesis & Relationship & Beta & SE & t-value & Decision \\
\hline H1 & Learning Styles -> Auditory & 0.82 & 0.04 & 21.75 & $\begin{array}{c}\text { Fail to Reject } \\
\text { (Supported) }\end{array}$ \\
\hline H2 & Learning Styles -> Phrase & 0.67 & 0.08 & 8.51 & $\begin{array}{c}\text { Fail to Reject } \\
\text { (Supported) }\end{array}$ \\
\hline H3 & Learning Styles -> Tactile & 0.77 & 0.07 & 10.83 & $\begin{array}{c}\text { Fail to Reject } \\
\text { (Supported) }\end{array}$ \\
\hline H4 & $\begin{array}{l}\text { Learning Styles -> } \\
\text { Kinesthetic }\end{array}$ & 0.76 & 0.05 & 16.66 & $\begin{array}{c}\text { Fail to Reject } \\
\text { (Supported) }\end{array}$ \\
\hline
\end{tabular}

Published By:

Blue Eyes Intelligence Engineering \& Sciences Publication

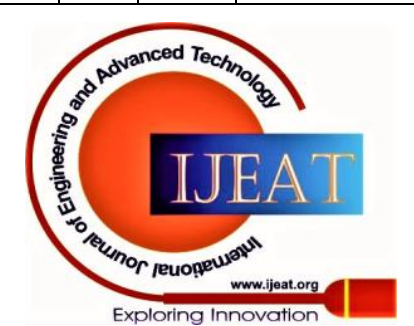




\begin{tabular}{|c|l|c|c|c|c|}
\hline H5 & Learning Styles -> Visual & 0.87 & 0.03 & 30.63 & $\begin{array}{c}\text { Fail to Reject } \\
\text { (Supported) }\end{array}$ \\
\hline H6 & Learning Styles -> Group & 0.74 & 0.06 & 12.32 & $\begin{array}{c}\text { Fail to Reject } \\
\text { (Supported) }\end{array}$ \\
\hline H7 & Learning Styles -> Individual & 0.49 & 0.09 & 5.70 & $\begin{array}{c}\text { Fail to Reject } \\
\text { (Supported) }\end{array}$ \\
\hline H8 & $\begin{array}{l}\text { Meaningful Learning -> } \\
\text { Objective }\end{array}$ & 0.73 & 0.06 & 11.75 & $\begin{array}{c}\text { Fail to Reject } \\
\text { (Supported) }\end{array}$ \\
\hline H9 & $\begin{array}{l}\text { Meaningful Learning -> } \\
\text { Active }\end{array}$ & 0.71 & 0.07 & 10.43 & $\begin{array}{c}\text { Fail to Reject } \\
\text { (Supported) }\end{array}$ \\
\hline H10 & $\begin{array}{l}\text { Meaningful Learning -> } \\
\text { Authentic }\end{array}$ & 0.75 & 0.05 & 13.66 & $\begin{array}{c}\text { Fail to Reject } \\
\text { (Supported) }\end{array}$ \\
\hline H11 & $\begin{array}{l}\text { Meaningful Learning -> } \\
\text { Cooperative }\end{array}$ & 0.48 & 0.12 & 4.03 & $\begin{array}{c}\text { Fail to Reject } \\
\text { (Supported) }\end{array}$ \\
\hline H12 & $\begin{array}{l}\text { Meaningful Learning -> } \\
\text { Constructive }\end{array}$ & 0.64 & 0.08 & 7.72 & $\begin{array}{c}\text { Fail to Reject } \\
\text { (Supported) }\end{array}$ \\
\hline H13 & $\begin{array}{l}\text { Learning Styles -> } \\
\text { Meaningful Learning }\end{array}$ & 0.36 & 0.12 & 3.07 & $\begin{array}{c}\text { Fail to Reject } \\
\text { (Supported) }\end{array}$ \\
\hline
\end{tabular}

\section{DISCUSSION}

This study supports the views of previous research [26] regarding the influence of independent variables of learning styles on the perceived extent of meaningful learning among Orang Asli students. The study employ partial least square (PLS) technique in hypothesis testing. This article also examined the appropriateness of the measures of the instruments used. This was achieved by looking at the validity and reliability carried out using a PLS approach. The result showed that the convergent and discriminant validity of the measures used was supported. The next step was to assess the reliability of the measures by looking at the composite reliability (CR) values. CR values complied with the criteria set up by experts [21]. Thus, the measures in the model were deemed reliable. The findings showed that, learning styles of Orang Asli students reflect the aspects of auditory, phrase, tactile, kinesthetic, visual, group and individual. They learned better when teaching and learning incorporated their preference learning styles [27] [28]. The Orang Asli students' learning became more meaningful when the teaching and learning process was carried out via storytelling, and lecture [29]. They could memorize better through reading aloud or moving their lips while reading, especially when they learned new things. Orang Asli students with auditory learning styles benefitted when they were involved in group discussion, where they tended to listen deeply to what others were discussing [30]. They also preferred learning in groups rather than individually. This refers to the sociology learning style. These students could learn more effectively within a group of two or more friends [31] [32]. Stimulus obtained in a group helped them to better learn and understand new information. They were more successful in completing tasks if they worked in a team. The phrase attribute refers to the number of words used in the teaching and learning process. Orang Asli students preferred notes or activities which contained a smal number of words to deliver the information. When a sentence consisted of too many words, Orang Asli students found it to be difficult to understand [17]. Reading and understanding longer sentences seemed to be difficult for those with lower literacy. Thus, the selection of phrases that consisted of two to three words was appropriate for Orang Asli students. Orang Asli students learned better when they practiced what they had studied [33] [34]. Their learning was more meaningful when they had the opportunity to practice what they had learned by using suitable equipment and materials [31]. Writing notes or instructions could help them remember the information, while physical involvement in classroom activities helped them understand new information. Orang Asli gained a better understanding when they practiced the information received, and when they were allowed to move around in the classroom, such as walking from one place to another in class [29]. [35] stated that, students with kinesthetic learning styles could learn better through experience, Moreover, they could remember information better when they actively participated in the activities, and learned by doing things. Studies showed that students who were taught by associating Orang Asli students in their experience increased the level of their academic achievement [36] [37] [38]. Orang Asli students learn effectively with pictures, charts, and graphs [39]. Students with a visual learning style easily remember and better understand information and instructions when it is read. They do not require further explanation, and prefer to study on their own. This is associated with second sociology learning which is categorized as individual. Students prefer to learn on their own, and can learn better when alone. They can think better, find it is easy to understand new materials, and demonstrate high performance when studying alone [40]. This learning style provides an opportunity for students to show their creativity and skills without being influenced by their peers. Besides the Orang Asli students' learning styles, the PLS analysis also showed that meaningful learning among Orang Asli students reflects five attributes. There are objective, active, authentic, constructive, and cooperative. These five attribute are the indicators which identify how meaningful learning occurs among these students. Objectives are the main focus during class session. The explanation of the objectives by the teacher guides the students through the flow of the lesson on that day. The same explanation should be provided while teaching Orang Asli students. Teachers should inform the student of the learning objective and the output at the end of the class session. Usually this process is conducted verbally by the teachers, and students listen and understand. This relates to the auditory learning styles of Orang Asli students who tend to listen to what their teachers are saying [41]. This shows that the auditory learning style contributes to meaningful learning in Orang Asli students. Students who are gaining from meaningful learning are active in constructing their own learning and develop a flexible framework that can be applied to various problems [42]. In addition, active learning occurs when the learning process happens through interaction and manipulation of tools [43]. Active learning refers to related course activities that students in a classroom take part in besides watching, listening and copying notes [44]. Meaningful learning requires that each student actively constructs his or her own knowledge. According to [45], this new knowledge is built on the basis of prior knowledge, beliefs and prejudices where new elements are incorporated by learning basic prior knowledge to build a framework of effective and comprehensive concept for their domain. The attributes of kinesthetic and tactile learning style demonstrated by Orang Asli students contribute to this meaningful learning. According to [43] [46], authentic is something that reflects the complex thoughts and ideas that depend on the context in which it occurs and brings a particular purpose. In other words, authentic learning happens when the activities undertaken are related to real life situations [47] [48]. According to [49], a hybrid e-training environment exposes students to the creative development of knowledge.

Published By:

Blue Eyes Intelligence Engineering

\& Sciences Publication

(C) Convriaht: All riahts reserved

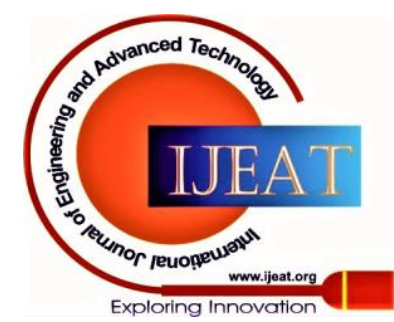




\section{The Impact of Orang Asli Students' Learning Styles on Their Achievement of Meaningful Learning}

In the present study, Orang Asli students experienced authentic meaningful learning when the process of learning was associated with the environment around them. The environment has a high impact on the teaching and learning process of Orang Asli students which contributes to understanding the information, effectively and meaningfully [26]. This process was conducted through the implementation of the visual and auditory learning styles attribute in conjunction with their environment. As an example, a traditional dance video was shown during a class session as it conveyed a message related to the study. The construction of knowledge brings meaningful learning when students take a new experience and relate it to prior knowledge [50]. Through this process students are able to create a mental model to understand the world [46] [48]. Students need to reflect upon the events, observations and their interpretation to gain a meaningful learning experience [46] [50]. The phrase learning style contributes to the process of achieving meaningful learning [17]. This is because, when few words are used in modules or activities, students with low reading skills can understand what they are being taught. Such Orang Asli students can recall past experience and relate it to what they learned which contributes to the creation of new knowledge. Cooperative learning relates to the involvement of students working in pairs or in a group [43] [51]. This learning process is relevant to Orang Asli students who favor the group learning style. According to [52], Orang Asli students who complete assignments in groups can produce work of a good quality. Orang Asli students have different levels of the same capabilities, and this is how they get things done together. They mutually help each other to achieve a common goal [53]. Thus, cooperative learning provides opportunities for students to engage in discussion, take charge of their learning, and engage in critical thinking [54]. The findings also confirmed the views of previous research that learning styles have an impact towards the meaningful learning of Orang Asli students [55] [56]. This supports findings from [26] [57] [58]. Orang Asli students have a different genre of learning and behaviors compared to non-Orang Asli students [16]. This may be observed during literacy lessons. Orang Asli students can understand better if they are taught using a story telling approach, compared to mainstream students in a formal education setting [26] [58]. Thus, teachers should take into account these differences in order to ensure that Orang Asli students understand better what they are being taught. Consequently, the process of teaching and learning which is based on the needs of mainstream students will be less effective when applied to Orang Asli students [13]. Thus, teachers should identify the Orang Asli students' learning styles, in order for them to understand the lesson more easily [26].

\section{CONCLUSION}

The application of learning styles in the process of teaching and learning for Orang Asli students contributes to their meaningful learning experience. Meaningful learning is essential in making sure that such students comprehend and use the knowledge and information they have learned for their daily life and future. Therefore, learning styles are important to enhance the level of meaningful learning among Orang Asli students. Findings also implied in term of practical and theoretical aspects. The practical implication is referring to preparing the process guideline for future education implementation among the Orang Asli students as well as preparing findings framework as a guideline and reference for future research on implementing education which involving Orang Asli students. On the other hand, the theoretical implication shown meaningful learning and learning styles provide added value to productive education environment among Orang Asli students were discovered.

\section{ACKNOWLEDGMENT}

Highest gratitude to GG-2019-066 and GG-2019-068 Grants for sponsoring the study. The author would like to thank Orang Asli community in Selangor who was involved for their cooperation and feedback to ensure the research fulfill the aim.

\section{REFERENCES}

1. Noraida Harun \& Noor Ashikin Hamid, Akta Orang Asli 1954 (Akta 134): Sejauh Mana Melindungi Hak Orang Asli: Satu Kajian Perbandingan. 2010 https://www.unisza.edu.my/perpustakaan/images/IDC/orang\%20asli.p df

2. $\bar{K}$ KLW. Kemajuan Perlaksanaan Pelan Tindakan Pembangunan Pendidikan Masyarakat Orang Asli Kawasan Pinggir Bagi Tahun 2003 Hingga Tahun 2006: Isu dan Cadangan Penambahbaikan. Kertas dibentangkan di Majlis Penasihat Kebangsaan Pembangunan Masyarakat Orang Asli, Bil 1/2007. Putrajaya (unpublished).

3. Adliza Othman, Hubungan Etnik. 2007 http://www.slideshare.net/finaz_fbi/hubunganetnik

4. Abdul Razaq Ahmad \& Zalizan Mohd Jelas, Masyarakat Orang Asli: Perspektif Pendidikan dan Sosiobudaya. Penerbit UKM, Bangi, 2009 pp.47-58.

5. Alicia, B. \& Ruth, W, Indigenous People and E-nabling Technologies: An Analysis of Recent Experiences in Northern and Central Australia. Kulumun. Indigenous Online Journal, 2011, 1, 1-14.

6. Batchelor, J. \& Lautenbach, G, Cultivating Lifelong Learning: Pre-service Teachers and their MOOCs. IST-Africa Conference, Lilongwe, Malawi, 2015. http://dx.doi.org/10.1109/ISTAFRICA.2015.7190557

7. Chea, C. C., Halim, N. A., \& Omar, N. A, What is the Role of Lifelong Learning in Relation to Economic Growth in Malaysia? Malaysian Journal of Distance Education, 2013, 15(1), 13-27.

8. Sharma, P, A Study of the Impact of Ict Tools on Achievement of Students in Learning Educational Technology. International Journal of Research (IJR), 2014, 1(6), 244-250.

9. Arora, N. \& Arora, K., V, Forward: Life-Long Education for the Global Workforce in the Socio-Engineering Age. Pertanika Journal of Social Sciences \& Humanities, 2015, 23(1), iii-xxvi.

10. Covallo, L., M,. A, Meaningful learning, Reasoning Ability, and Students' Understanding and Problem Solving of Topics in Genetics. Journal of Research in Science Teaching, 1996, 33(6), 626-656.

11. Alberta Education, Learning Strategies for Aboriginals Students: Opportunities to make Learning Meaningful, 2005. https://education.alberta.ca/teachers/resources/cross/ourwordsourways .aspx

12. McLarty, L. \& Moran, R, Engaging All Young People in Meaningful Learning After 16: A Qualitative Study. Equality and Human Rights Commission, 2009.

13. Burridge, N., Whalan, F., \& Vaughan, K, Indigenous Education a Learning Journey for Teachers, Schools and Communities. Sense publishers, 2012

14. Wan Hafizi Wan Hanafi, Shaharuddin Ahmad, \& Noraziah Ali, Faktor Budaya dan Persekitaran dalam Prestasi Pendidikan Anak Orang Asli Malaysia: Kajian Kes di Kelantan. GEOGRAFIA Online Malaysian Journal of Society and Space, 2014, 10(5), 107-122.

15. Bemen Win Keong Wong \& Christopher Perumal, The Issues of Teaching and Learning in the Primary School of Orang Asli: A Case Study of Pos Senderut, Kuala Lipis, Pahang. BIMP-EAGA Conference, pp 1-9, 2012.

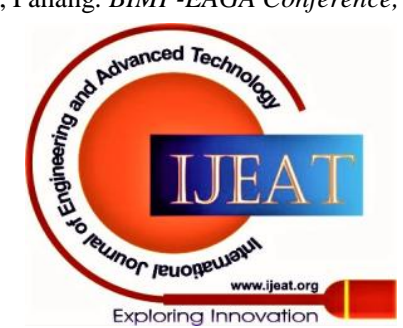


16. Mohamad Johdi Salleh, Norul Khairiah Idris, Nur Awanis Abd Aziz, Nurul Huda Yusuf \& Siti Aisyah Hashim, Kajian Terhadap Kesedaran Pendidikan di Kalangan Masyarakat Orang Asli. Persidangan Kebangsaan Pendidikan Luar Bandar, 2009, 1-15.

17. Sharma, P, A Study of the Impact of Ict Tools on Achievement of Students in Learning Educational Technology. International Journal of Research (IJR), 2014, 1(6), 244-250.

18. Hair, J. F., Ringle, C. M., \& Sarstedt, M. PLS-SEM: Indeed a Silver Bullet. The Journal of Marketing Theory and Practice, 2011, 19(2), 139-152.

19. Hair Jr, J. F., Hult, G. T. M., Ringle, C., \& Sarstedt, M, A Primer on Partial Least Squares Structural Equation Modeling (PLS-SEM): SAGE Publications, Incorporated, 2014

20. Hulland, J, Use of Partial Least Square (PLS) in Strategic Management Research: A Review of Four Recent Studies. Strategic Management Journal, 1999, 20(2), 195-204.

21. Hair, J. F., Black, W. C., Babin, B. J., \& Anderson, R. E, Multivariate Data Analysis. Upper Saddle River, NJ: Prentice Hall, 2010.

22. Sekaran, U., \& Bougie, R, Research Methods for Business: A Skill Building Approach. Wiley, UK, 2010.

23. Barclay DW, Thompson R, Higgins C, The Partial Least Squares (PLS) Approach To Causal Modeling: Personal Computer Adoption And Use An Illustration. Technol Stud, 1995, 2(2), 285-309.

24. Ramayah, T., Lee, J. W. C., \& In, J. B. C, Network Collaboration and Performance in the Tourism Sector. Springer, 2011, 5, 411-428. http://dx.doi:10.1007/s11628-011-0120-z

25. Compeau DR, Higgins CA, Huff S, Social Cognitive Theory and Individual Reactions to Computing Technology: A Longitudinal-Study. MISQ, 1999, 23(2), 145-158

26. Perso, T. F, Cultural Responsiveness and School Education: With Particular Focus on Australia's First Peoples; A Review \& Synthesis Of The Literature. Menzies School of Health Research, Centre for Child Development and Education, Darwin Northern Territory, 2012.

27. Jabari, M., A., D, New learning styles models in adaptive educational process. Jurnal Pendidikan Sains, 2020, 8(1): 1-6

28. Isda, D., I., Purwati, Baihaqi, \& Nuramalina. A study of efl students' learning styles in english classroom interaction. Proceedings of the 2nd English Education International Conference (EEIC) in conjunction with the 9th Annual International Conference (AIC), Universitas Syiah Kuala, September 18-19, 2016, Banda Aceh, Indonesia.

29. Gilakjani, P., A, Visual, Auditory, Kinaesthetic Learning Styles and Their Impacts on English Language Teaching. Journal of Studies in Education, 2012, 2(1), 104-113

30. Kitchenham, A, Indigenous Learning Preferences and Interactive Technologies. The Australian Journal of Indigenous Education, 2017, 46(1): 71-79.

31. Zokaee, S., Zaferanieh, E., \& Naseri, M, On the Impacts of Perceptual Learning Style and Gender on Iranian Undergraduate EFL Learners' Choice of Vocabulary Learning Strategies. English Language Teaching, 2013, 5(9), 138-143

32. Mat Salleh, N., Abdul Karim, A., Mat Deli, M., Abdul Manaf, S., Jz Nun Ramlan, N., Hamdan, A, An evaluation of content creation for personalised learning using digital ICT literacy moduel aming aboriginal students (mLICT-OA). Turkish Online Journal of Distance Education, 2019, 20(3): 41-58

33. Ghada, S., Rima, B., Nahla Nola, B., \& Mona, M, A Match or a Mismatch between Student and Teacher Learning Style Preferences. International Journal of English Linguistics, 2011, 1(1), 162-172

34. Espiritu, Jo \& Romualdo, Edilberta, Learning Style Preferences and Performance in Tle (Crop Production) Among Grade VII Indigenous Learners in Baytan, Botolan, Zambales, 2018. Available at SSRN: https://ssrn.com/abstract=3482404 or http://dx.doi.org/10.213 9/ssrn.3482404

35. Rini, S., D, Adisyahputra, Sigit, V., D, Boosting Student Critica Thinking Ability through Project Based Learning, Motivation and Visual, Auditory, Kinesthetic Learning Style: A study on Ecosystem Topic. Universal Journal of Educational Research, 2020, 8(4A): 37-44

36. Au, K., H., \& Kawakami, A. J,. Cultural Congruence in Instruction. In E. R. Hollins, J. E. King, \& W. C. Hayman (Eds.), Teaching diverse populations: Formulating a knowledge base (pp. 5-23). Albany: State University of New York Press, 1994.

37. Gay, G. Culturally responsive schooling: theory, research, and practice. Teachers College Press, N.Y, 2000.

38. Hollins, E. R, Culture in School Learning; Revealing the Deep Meaning. Mahwah, NJ: Lawrence Erlbaum, 1996.

39. Vaishnav, S., R, Learning Style And Academic Achievement Of Secondary School Students. Voice Of Research, 2013, 1(4), 1-4
40. Hughes, P., \& More, J., A, Aboriginal Ways of Learning and Learning Styles. Australian Association for Research in Education, 1997.

41. More, A, Learning Styles of Native Americans and Asians. Paper presented to American Psychological Association Conference, Bostan, 1990.

42. Hannafin, M. \& Land, S, The Foundations and Assumptions of Technology-Enhanced Student Centered Learning Environments. Instructional Science, 1997, 25(3), 167-202.

43. Chai, C. S., Tan, S. C., Wong, M. F. R., \& Ong, K. K. A, Collaborative Learning and the Use of ICT in Singapore Schools - 2 Case Studies. Paper presented at the 15th Global Chinese Conference on Computers in Education, 2011.

44. Settles, B, Active Learning Literature Survey. Technical report, 2010.

45. Bransford J., Brown, A. \& Cocking, R, How People Learn. National Academy Press: Washington, D. C. 2002.

46. Jonassen, D.H., Peck, K.L. \& Wilson B.G, Learning with Technology: A Constructivist Perspective. NJ: Merrill Prentice Hall, 1999.

47. Jonassen, D. H. et al, Meaningful Learning with Technology. 3rd ed. Allyn \& Bacon. 2007.

48. Burnaford, E., G., April, A., \& Weiss, C, Renaissance in the Classroom: Arts Integration and Meaningful Learning. Chicago Arts Partnerships in Education, CAPE, 2013.

49. Isayeva, O., Shumylo, M., Khmilyar, I., Myskiv, I., Mylyk, O, Blended learning in higher medical education: principles and strategies of teaching foreign languages. Advanced Education, 2020, 14: 11-18

50. Hung, V.H.K., Keppell, M. \& Jong, M. S.Y, Learners as Producers: Using Project Based Learning to Enhance Meaningful Learning through Digital Video Production, 2004.

51. Sharan, Y, Cooperative Learning for Academic and Social Gains: valued pedagogy, problematic practice. European Journal of Education Research, Development And Policy, 2010, 45(2), 300-313

52. Vermon, P., A., Jackson, D., N., \& Messick, S, Cultural Influences on Patterns of Abilities in North America. In S., H., Irvine \& J., W., Berry, (Eds). Human abilities in cultural context, Cambridge University Press, Cambridge, 1998, 208-231

53. Melinda, D, Constructing Knowledge Together. Bern: Peter Lang 2008.

54. Totten, S., Sills, T., Digby, A., \& Russ, P, Cooperative Learning: A guide to Research. New York: Garland. 1991

55. Burgess, C., Tennent, C., Vass, G., Guenther, J. Lowe, K., \& Moodie, $\mathrm{N}$, A systematic review of pedagogies that support, engage and improve the educational outcomes of aboriginal students. The Australian educational researched, 2019, 46: 297-318

56. Mohd Nazri Abdul Rahman, Rosmah Abd Ghani, Khairul Azhar Jamaluddin, Hazlina Awang Lah, Anis Salwani Mahmood, Ismail Sulaiman, \& Wan Azani Mustafa, Development of Folktales Digita Story Module as Basic Literacy Learning for Indigenous Children in Rural Preschool. Journal of Physics, 2019, 1529.

57. Hughes, P., \& More, J., A, Aboriginal Ways of Learning and Learning Styles. Australian Association for Research in Education, 1997.

58. Sumathi, R, ‘A Pet Rabbit, Bunny': Teaching English to Orang Asl Children, an Indigenous community in Malaysia. IPEDR, 2013, 68(25), 145-151

\section{AUTHORS PROFILE}

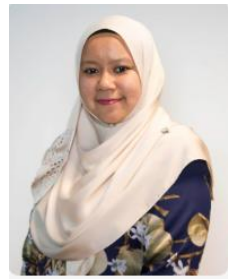

Intan Farahana Kamsin, Final year $\mathrm{PhD}$ Candidate in Education (Resources and Information Technology). She is currently working as a lecturer in School of Technology department at Asia Pacific University of Technology and Innovation (APU). Her research interests are: Internet of Things (IOT), e-learning, education among marginalized community, emerging technology for educational purposes, big data, and data analytics. She is a member of Malaysian Higher Education Research and Policy Development Organization (PenDaPaT)

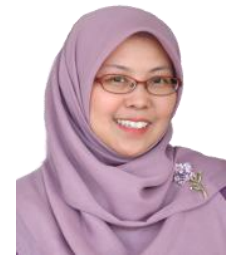

Fariza Khalid is a senior lecturer and the Head of Ph.D. Program at the Faculty of Education, Universiti Kebangsaan Malaysia. She received her $\mathrm{PhD}$ in the area of Instructional Technology from the University of Nottingham. Her current research areas are:

Published By:

Blue Eyes Intelligence Engineering \& Sciences Publication

(C) Convriaht: All riahts reserved

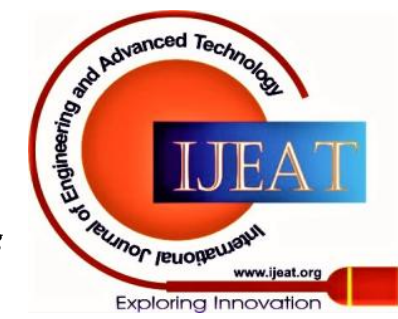


The Impact of Orang Asli Students' Learning Styles on Their Achievement of Meaningful Learning

online communities of practice, augmented reality for educational purposes, cybersecurity awareness and online and offline identities. She is also a life-member of Qualitative Research Association of Malaysia (QRAM), International Association for Development of Information Society (IADIS) and Informing Science Institute (ISI).

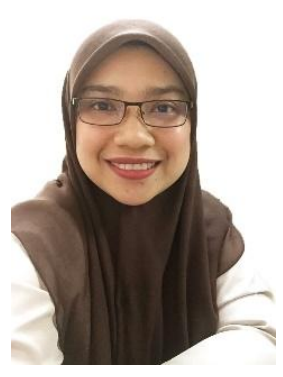

Nor Syazwani Mat Salleh, a senior lecturer at Faculty of Art, Computing \& Creative Industry, Sultan Idris Education University (UPSI). Completed her Ph.D at Faculty of Education, Universiti Kebangsaan Malaysia (UKM). She has a Bachelor Degree in Software Engineering from Universiti Tun Hussein Onn Malaysia (UTHM) and a Master in Computer Education from UKM. Her research interests are: Computer Education and Resources \& Information Technology, Personalized Learning, Art Education and Technology, Visual Communication and Graphic Design. She has published more than 15 articles in international journals, and resented her research in various national and international conferences.

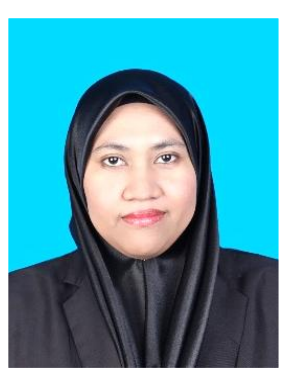

Analisa Hamdan holds a B. Ed. Information Technology (Honours) from Universiti Utara Malaysia (UUM) in 2007 and a Master of Education from Open University Malaysia (OUM) in 2019. She is currently a Ph.D. student of Instructional Technology Education from the Faculty of Human Development, Universiti Pendidikan Sultan Idris (UPSI), Malaysia. Her professional interests include mobile learning, personalized learning environments, information and communication technologies (ICT), educational technology, and e-learning. She has won with Gold \& Silver medals in the National University Carnival on E-Learning 2014 (NUCEL 2014).

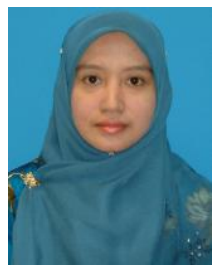

Siti Zuraida Abdul Manaf graduated from Education Faculty, Universiti Kebangsaan Malaysia in Research Information Technology (Phd). She is interested in research in technology system, Mapping, GIS (geography information system), big data, and mobile learning. 